\title{
On the Stability of Fuzzy Systems
}

\author{
M. A. L. Thathachar and Pramod Viswanath
}

\begin{abstract}
This paper studies the global asymptotic stability of a class of fuzzy systems. It demonstrates the equivalence of stability properties of fuzzy systems and linear time invariant (LTI) switching systems. A necessary condition and a sufficient condition for the stability of such systems are given, and it is shown that under the sufficient condition, a common Lyapunov function exists for the LTI subsystems. A particular case when the system matrices can be simultaneously transformed to normal matrices is shown to correspond to the existence of a common quadratic Lyapunov function. A constructive procedure to check the possibility of simultaneous transformation to normal matrices is provided.
\end{abstract}

Index Terms-Asymptotic stability, switching systems.

\section{INTRODUCTION}

$\mathbf{R}$ ECENTLY, fuzzy control is being used in many practical industrial applications. One of the first questions to be answered in this context is the stability of the fuzzy system. In recent literature, Tanaka and Sugeno [3], have provided a sufficient condition for the asymptotic stability of a fuzzy system in the sense of Lyapunov through the existence of a common Lyapunov function for all the subsystems. Tanaka and Sano [4] have extended this to robust stability in case of systems with premise-parameter uncertainty. The model of the fuzzy system, considered in these papers, is that proposed by Takagi and Sugeno [2], which can be shown to be equivalent in stability to a switching system with linear time invariant (LTI) subsystems. These switching systems turn out to be a particular class among linear time varying (LTV) systems. The classical theory of LTV systems is discussed in [8, Sec. 9], and recent advances in LTV systems are in [9]. Narendra and Balakrishnan [6] have provided a simple sufficient condition for the stability of the switching system.

This paper discusses some necessary and some sufficient conditions for global asymptotic stability of a fuzzy system. Section II defines the fuzzy system model and the model of the corresponding switching system and global asymptotic stability of these systems. Section III shows the equivalence of these two systems regarding stability, and further sections consider switching systems only. Section IV discusses the main results of this paper. A sufficient condition is provided for the asymptotic stability of the system. It is shown that when this condition is satisfied, there exists a common Lyapunov function for the subsystems. Section V deals with a special case which leads to simultaneous normalization of the system matrices. In this case, the subsystems have a common

Manuscript received August 25, 1995; revised January 30, 1996.

M. A. L. Thathachar is with the Indian Institute of Science, Bangalore, 560012 India.

P. Viswanath is with the Electrical Engineering and Computer Science Department, University of California, Berkeley, CA 94720 USA.

Publisher Item Identifier S 1063-6706(97)00037-4. quadratic Lyapunov function. Also, a constructional procedure is provided to check whether the theorem's premise is satisfied. An example illustrates the procedure. This result can be viewed as a generalization of the result in [6], a sufficient condition for stability of switching systems.

\section{NOTATIONS AND DEFINITIONS}

This section outlines the mathematical model of a free fuzzy system and that of the corresponding switching system. Stability of these systems in the asymptotic sense is also defined.

The Takagi and Sugeno [2] model for the fuzzy system is chosen. Let the system state vector at time instant $k$ be $\bar{x}(k)=\left[x_{1}(k) \ldots x_{n}(k)\right]^{T}$ where $x_{1}(k) \cdots x_{n}(k)$ are the state variables of the system at time instant $k$. Then the free fuzzy system is defined by the implications below

$$
\begin{aligned}
R^{i}: & \operatorname{IF}\left(x_{1}(k) \text { is } \mathcal{S}_{1}^{i}, \text { AND } \cdots \text { AND } x_{n}(k) \text { is } \mathcal{S}_{n}^{i}\right) \\
& \operatorname{THEN} \bar{x}(k+1)=A_{i} \bar{x}(k)
\end{aligned}
$$

for $i=1 \cdots N$. Here, $\mathcal{S}_{j}^{l}$ is the fuzzy set corresponding to the state variable $x_{j}$ and implication $R^{l} . A_{i} \in \mathcal{R}^{n \times n}, i=1 \cdots N$ are the system characteristic matrices. The truth value of the implication $R^{i}$ at time instant $k$ denoted by $w_{i}(k)$ is defined as

$$
w_{i}(k)=\wedge\left(\mu_{\mathcal{S}_{1}^{i}}\left(x_{1}(k)\right), \cdots, \mu_{\mathcal{S}_{n}^{i}}\left(x_{n}(k)\right)\right.
$$

where $\mu_{\mathcal{S}}(x)$ is the membership function value of the fuzzy set $\mathcal{S}$ at the position $x$ and $\wedge$ is an operator satisfying

$$
\min \left(l_{1}, \cdots, l_{n}\right) \geq \wedge\left(l_{1}, \cdots, l_{n}\right) \geq 0 .
$$

Usually $\wedge$ is taken to be the minimum operator which gives the minimum of its operands. Then, at instant $k$ the state vector is updated according to

$$
\begin{aligned}
\bar{x}(k+1) & =\frac{\left(\sum_{i=1}^{N} w_{i}(k) A_{i} \bar{x}(k)\right)}{\sum_{i=1}^{N} w_{i}(k)} \\
& =\sum_{i=1}^{N} \alpha_{i}(k) A_{i} \bar{x}(k) ; \quad \alpha_{i}(k)=\frac{w_{i}(k)}{\sum_{i=1}^{N} w_{i}(k)} .
\end{aligned}
$$

A fuzzy system is completely represented by the set of characteristic matrices $\mathcal{A}=\left[A_{1}, \cdots, A_{n}\right]$ and the fuzzy sets $\mathcal{S}_{j}^{l}, l=1, \cdots, N ; j=1, \cdots, n$. Corresponding to this free fuzzy system, a corresponding switching system is described below. 
The state update at time instant $k$ is given as

$$
\bar{x}(k+1)=A \bar{x}(k)
$$

where $A \in \mathcal{A}$ (i.e., it is one of the matrices $A_{1}, A_{2}, \cdots, A_{n}$ ). Also, define $\forall k \geq 1$

$$
\mathcal{A}_{k}=\underbrace{\mathcal{A} \times \mathcal{A} \times \cdots \times \mathcal{A}}_{k \text { times }}
$$

where the Cartesian product is defined to be the multiplication of matrices in the same order. The following are definitions of global asymptotic stability of these systems.

Definition 1) The fuzzy system described in (2) is globally asymptotically stable if

$$
\bar{x}(k) \rightarrow 0 \quad \text { as } \quad k \rightarrow \infty
$$

or, equivalently, there exists $\|\cdot\|$, a norm on $\mathcal{R}^{n}$

$$
\|\bar{x}(k)\| \rightarrow 0 \quad \text { as } \quad k \rightarrow \infty
$$

for all initial values $x(0) \in \mathcal{R}^{n}$ and for all possible fuzzy sets $\mathcal{S}_{i}^{j}, \forall i=1 \cdots N, \forall j=$ $1 \cdots n$.

Definition 2) The switching system described in (3) is globally asymptotically stable if

$$
\begin{aligned}
& \bar{x}(k+1)=A(k) \bar{x}(0) \rightarrow 0 \\
& \text { as } \quad k \rightarrow \infty ; \quad \forall \bar{x}(0) \in \mathcal{R}^{n}
\end{aligned}
$$

where $A(k) \in \mathcal{A}_{k}$. Equivalently

$$
A(k) \rightarrow 0 \quad \text { as } k \rightarrow \infty ; \quad A(k) \in \mathcal{A}_{k} .
$$

For any matrix $A \in \mathcal{R}^{n}$, let $\rho(A)$ be the spectral radius of $A$ (i.e., the largest magnitude of the eigen values of $A$ ). Let $\rho\left(\mathcal{A}_{k}\right)$ be defined as

$$
\rho\left(\mathcal{A}_{k}\right)=\max \left\{\rho(A): A \in \mathcal{A}_{k}\right\} .
$$

Let $\|\cdot\|$ be a matrix norm on $\mathcal{R}^{n}$ and $\sigma(A)$ be the largest singular value of $A$. Then,

$$
\begin{aligned}
\sigma(A) & =\sqrt{\rho\left(A^{T} A\right)} \\
& =\|A\|_{\mathrm{sp}}
\end{aligned}
$$

where $\|\cdot\|_{\mathrm{sp}}$ is the spectral norm on a matrix. Also, let $\sigma\left(\mathcal{A}_{k}\right)=\max \left\{\sigma(A): A \in \mathcal{A}_{k}\right\}$. Let $(A)_{i j}$ represent the element $a_{i j}$ where $A=\left[a_{i j}\right]$. Let $A^{*}$ denote the conjugate transpose of $A$.

\section{Equivalence OF THE Stability OF SWITCHING AND FuZZY SYSTEMS}

This section illustrates the equivalence of the stability of a fuzzy system and its corresponding switching system. A necessary condition for stability of either of these systems is also given. The following theorem illustrates some equivalent statements about the stability of the switching system. The proof might be hidden in some textbook; we give it here for completeness.
Theorem 3.1: The following are equivalent:

1) the switching system in (3) is globally asymptotically stable as in Definition 2;

2) $\sigma\left(\mathcal{A}_{k}\right) \rightarrow 0$ as $k \rightarrow \infty$;

3) $\rho\left(\mathcal{A}_{k}\right) \rightarrow 0$ as $k \rightarrow \infty$.

Proof: $(1) \Rightarrow(2)$

By (6), $A \overline{(k) \rightarrow 0}$ as $k \rightarrow \infty ; A(k) \in \mathcal{A}_{k}$. By (7), $\sigma(\cdot)$ is a matrix norm and, hence

$$
\sigma(A(k)) \rightarrow 0 \quad \text { as } \quad k \rightarrow \infty ; \quad \forall A(k) \in \mathcal{A}_{k} .
$$

In particular, $\max _{A(k) \in \mathcal{A}_{k}}[\sigma(A(k))] \rightarrow 0$ as $k \rightarrow \infty$. Hence

$$
\sigma\left(\mathcal{A}_{k}\right) \rightarrow 0 ; \quad \text { as } \quad k \rightarrow \infty
$$

$\frac{(2) \rightarrow(3)}{\text { Now }}$

$$
\begin{aligned}
\sigma\left(\mathcal{A}_{k}\right) & =\max \left\{\sigma(A): A \in \mathcal{A}_{k}\right. \\
& \geq \max \left\{\rho(A): A \in \mathcal{A}_{k}\right\}
\end{aligned}
$$

since $\|A\| \geq \rho(A)$ from [1, Theorem 5.6.9]. Hence

$$
\sigma\left(\mathcal{A}_{k}\right) \geq \rho\left(\mathcal{A}_{k}\right) \geq 0 ; \quad \forall k \geq 1
$$

and $\rho\left(\mathcal{A}_{k}\right) \rightarrow 0$ as $k \rightarrow \infty$.

$$
(3) \rightarrow(1)
$$

From [7, Sec. 7.2], $\rho(A)$ is a continuous function of the elements of $A$. Since $\rho\left(\mathcal{A}_{k}\right) \rightarrow 0$ as $k \rightarrow \infty$, we have

$$
\rho(A(k)) \rightarrow 0 \quad \text { as } \quad k \rightarrow \infty ; \quad \forall A(k) \in \mathcal{A}_{k} .
$$

Since $\rho(A(k))$ is a continuous function of $A(k)$

$$
A(k) \rightarrow 0 \quad \text { as } \quad k \rightarrow \infty ; \quad \forall A(k) \in \mathcal{A}_{k}
$$

hence arriving at (6), which is the required result.

The following theorem illustrates the equivalence of stability of a fuzzy system and that of the corresponding switching system.

Theorem 3.2: A necessary and sufficient condition for the stability as in Definition 1 of fuzzy system (2) is that the corresponding switching system (3) be stable, as in Definition 2.

Proof necessity: The fuzzy system in (2) degenerates into the switching system when $w_{i}=1$ or $0, i=1 \cdots N$, and $\sum_{i=1}^{N} w_{i}=1$. Thus, the switching system should necessarily be stable.

Sufficiency: Let the switching system represented by $\mathcal{A}=\left\{A_{1}, \cdots, A_{n}\right\}$ be stable as in Definition 2. The proof that fuzzy system is also stable uses the fact that given any two sequences $a_{k}$ and $b_{k}$ in $R^{n}$, such that

$$
\begin{aligned}
& \left\|a_{k}\right\| \geq\left\|b_{k}\right\| \forall k \geq K \text { and } a_{k} \rightarrow 0 \text { as } k \rightarrow \infty \\
& \text { implies } b_{k} \rightarrow 0 \text { as } k \rightarrow \infty .
\end{aligned}
$$


Now, the fuzzy system is stable if $\forall \bar{x}(0) \in \mathcal{R}^{n}$ and all fuzzy sets $S_{i}^{j}, i=1 \cdots N, j=1 \cdots n$, as $k \rightarrow \infty$

$$
\begin{aligned}
\left(\sum_{i_{k}=1}^{N} \alpha_{i_{k}}(k-1) A_{i_{k}}\right) \cdots\left(\sum_{i_{1}=1}^{N} \alpha_{i_{1}}(0) A_{i_{1}}\right) \bar{x}(0) & \rightarrow 0 \\
\text { i.e., }\left(\sum_{i_{k}=1}^{N} \alpha_{i_{k}}(k-1) A_{i_{k}}\right) \cdots\left(\sum_{i_{1}=1}^{N} \alpha_{i_{1}}(0) A_{i_{1}}\right) & \rightarrow 0 \\
\text { i.e. } \sum_{i_{1} \cdots i_{k}=1 \cdots N} \alpha_{i_{k}}(k-1) \cdots \alpha_{i_{1}}(0) A_{i_{k}} \cdots A_{i_{1}} & \rightarrow 0 .
\end{aligned}
$$

Now

$$
\begin{aligned}
\| & \sum_{i_{1} \cdots i_{k}=1 \cdots N} \alpha_{i_{1}}(0) \cdots \alpha_{i_{k}}(k-1) A_{i_{k}} \cdots A_{i_{1}} \|_{\mathrm{sp}} \\
& \leq \sum_{i_{1} \cdots i_{k}=1 \cdots N} \alpha_{i_{1}}(0) \cdots \alpha_{i_{k}}(k-1)\left\|A_{i_{k}} \cdots A_{i_{1}}\right\|_{\mathrm{sp}} \\
= & \sum_{i_{1} \cdots i_{k}=1 \cdots N} \alpha_{i_{1}}(0) \cdots \alpha_{i_{k}}(k-1) \sigma\left(A_{i_{k}} \cdots A_{i_{1}}\right) \\
\leq & \sum_{i_{1}, \cdots, i_{k}=1 \cdots N} \alpha_{i_{1}}(0) \cdots \alpha_{i_{k}}(k-1) \\
& \times \max _{i_{1}, i_{2}, \cdots, i_{k}}\left\{\sigma\left(A_{i_{k}} A_{i_{k-1}} \cdots A_{i_{1}}\right)\right\} \\
= & \sigma\left(\mathcal{A}_{k}\right) \sum_{i_{1}, \cdots, i_{k}=1 \cdots N} \alpha_{i_{1}}(0) \cdots \alpha_{i_{k}}(k-1) \\
= & \sigma\left(\mathcal{A}_{k}\right)
\end{aligned}
$$

since $\Sigma_{i_{j}=1 \cdots N} \alpha_{i_{j}}=1 ; \forall j=1 \cdots k$.

Now, since the switching system is stable, by Theorem 3.1 we have $\sigma\left(\mathcal{A}_{k}\right) \rightarrow 0$ as $k \rightarrow \infty$. From (8), left-hand side $\rightarrow 0$ and the condition for stability (4) is satisfied. Hence, the fuzzy system too is stable, as in Definition 1.

From now on only the stability of the switching system is mentioned. The following theorem provides a necessary condition for the stability of the switching system.

Theorem 3.3: A necessary condition for stability (as in Definition 2) of the switching system in (3) is that every finite product sequence of the matrices in $\mathcal{A}$ be stable, i.e., their spectral radius is less than one. Equivalently

$$
\rho\left(\mathcal{A}_{k}\right)<1 ; \quad \forall k \geq 1 .
$$

Proof: Suppose not. Then, $\exists A_{l}=A_{i_{1}} A_{i_{2}} \cdots A_{i_{l}} \in \mathcal{A}_{l}$ such that $\rho\left(A_{i_{1}} A_{i_{2}} \cdots A_{i_{l}}\right) \geq 1$. Then, consider the switching sequence

$$
\bar{x}(k+l)=A_{i_{1}} A_{i_{2}} \cdots A_{i_{l}} \bar{x}(k) .
$$

Then, $\bar{x}(k+m * l)=A_{l}^{m} \bar{x}(k)$. Clearly, $\bar{x}(k) \nrightarrow 0$ as $k \rightarrow \infty$ since $\rho\left(A_{l}\right) \geq 1$. Hence, every finite product sequence of the matrices has to be stable.

Comment 1: As an immediate consequence of the preceding theorem, the matrices $A_{1}, A_{2}, \cdots, A_{n}$ should, themselves, be necessarily stable (i.e., $\rho\left(A_{i}\right)<1 \forall i=1 \cdots N$ ).

Comment 2: Also, the result expressed in [3, Theorem 4.3], a necessary condition for stability that the matrices be pairwise stable, is evident as a special case of Theorem 3.3 in which $\rho\left(\mathcal{A}_{2}\right)$ is considered.

\section{A SUFFICIENT CONDITION FOR STABILITY}

The following Theorem 4.1 provides a sufficient condition for stability of (3). Theorem 4.2 shows that when the condition in Theorem 4.1 is satisfied, there exists a common Lyapunov function $V(\bar{x})$ for all the system matrices in $\mathcal{A}$. However, this common $V(\bar{x})$ is not necessarily quadratic.

Theorem 4.1 A sufficient condition for stability, as in Definition 2 of the switching system (3), is that there exists a similarity transformation $S \in R^{n \times n}$ and a matrix norm $\|\cdot\|$ such that

$$
\left\|S^{-1} A S\right\|<1 ; \quad \forall A \in \mathcal{A} \text {. }
$$

Proof: Let

$$
\eta=\max \left\{\left\|S^{-1} A S\right\| ; \quad \forall A \in \mathcal{A}\right\}<1 .
$$

Let

$$
A(k) \in \mathcal{A}_{k}=A_{i_{1}} A_{i_{2}} \cdots A_{i_{k}} .
$$

Also, let $S$ be the similarity transformation. Now $\forall A(k) \in \mathcal{A}_{k}$.

$$
\begin{aligned}
\rho(A(k)) & =\rho\left(A_{i_{1}} A_{i_{2}} \cdots A_{i_{k}}\right) \\
& =\rho\left(S^{-1} A_{i_{1}} A_{i_{2}} \cdots A_{i_{k}} S\right) \\
& \leq\left\|S^{-1} A_{i_{1}} A_{i_{2}} \cdots A_{i_{k}} S\right\| \\
& \quad \text { from Theorem 5.6.9 in }[1] \\
& \leq\left\|S^{-1} A_{i_{1}} S\right\| \cdot\left\|S^{-1} A_{i_{2}} S\right\| \cdots\left\|S^{-1} A_{i_{k}} S\right\| \\
& \leq \eta^{k} \text { from (11) and.(12) }
\end{aligned}
$$

Hence, $\rho\left(\mathcal{A}_{k}\right) \leq \eta^{k} ; \eta<1$ and $\rho\left(\mathcal{A}_{k}\right) \rightarrow 0$ as $k \rightarrow \infty$. Hence, the switching system is asymptotically stable.

Theorem 4.2: If (10) in Theorem 4.1 is satisfied, and the norm in (10) is an induced norm, then there exists a common Lyapunov function for all the system matrices in $\mathcal{A}$, i.e., there exists a function $V: R^{n} \rightarrow R^{+}$such that $\forall A \in \mathcal{A}$ :

- $V(\bar{x}(k))>0, \bar{x}(k) \neq 0$.

- $\Delta V(\bar{x}(k))=V(A \bar{x}(k))-V(\bar{x}(k))<0 \forall k \geq 0$.

Proof: Let the premise in Theorem 4.1 be satisfied and $S$ be the similarity transformation. Let $Q=S^{-1}$. Define $V(\bar{x}(k))=\|Q \bar{x}(k)\|^{2}$ where the vector norm here induces the matrix norm in (10). Now, since $S$ is nonsingular, $V(\bar{x}(k)) \neq$ 0 when $\bar{x}(k) \neq 0$. Now, $\forall A \in \mathcal{A}$ and $\bar{x}(k) \neq 0$,

$$
\begin{aligned}
\Delta V(\bar{x}(k)) & =\|Q \bar{x}(k+1)\|^{2}-\|Q \bar{x}(k)\|^{2} \\
& =\|Q A \bar{x}(k)\|^{2}-\|Q \bar{x}(k)\|^{2} \\
& =\|Q A S \bar{y}(k)\|^{2}-\|\bar{y}(k)\|^{2} \\
& \quad \text { where } \bar{y}(k)=Q \bar{x}(k) \\
& \leq\left\|S^{-1} A S\right\|^{2}\|\bar{y}(k)\|^{2}-\|\bar{y}(k)\|^{2} \\
& <0 \text { since } \bar{x}(k) \neq 0 \text { and from. }(10)
\end{aligned}
$$

This function is the common Lyapunov function.

However, this common Lyapunov function need not be quadratic, i.e., $V(\bar{x}(k))$ need not be of the form $\bar{x}(k)^{T} P \bar{x}(k)$ where $P$ is a positive definite matrix. 
Comment 1: A convenient matrix norm that can be used in (10) is the spectral norm $\|\cdot\|_{\mathrm{sp}}=\sigma(\cdot)$ and is induced from the Euclidean vector norm. (see $[1,5.5 .6])$. Hence, in particular, Theorem 4.1 reduces to the existence of a nonsingular $S$ such that $\sigma\left(S^{-1} A S\right)<1 ; \forall A \in \mathcal{A}$. In this case, the common Lyapunov function in Theorem 4.2 can be chosen to be quadratic. This is expressed in the following theorem.

Theorem 4.3: The satisfaction of (10) in Theorem 4.1 and the norm in (10) being the spectral norm (i.e., induced from the Euclidean vector norm) is a necessary and sufficient condition for the existence of a common quadratic Lyapunov function for all the system matrices in $\mathcal{A}$, i.e., $V(\bar{x})$ is of the form $\bar{x}^{T} P \bar{x}$ where $P$ is a positive definite matrix.

Proof necessity: Suppose there exists a common Lyapunov function $V(\bar{x})=\bar{x}^{T} P \bar{x}$ for all the system matrices $A \in \mathcal{A}$. Since $P$ is positive definite $\exists S$ nonsingular $\ni P=$ $Q^{T} Q$ where $Q=S^{-1}$. Then

$$
\begin{aligned}
V(\bar{x}(k)) & =\bar{x}(k)^{T} P \bar{x} \\
& =\bar{x}(k)^{T} Q^{T} Q \bar{x}(k) \\
& =\|Q \bar{x}(k)\|^{2}
\end{aligned}
$$

where $\|\cdot\|$ is the Euclidean norm.

Since $\Delta V(\bar{x}(k))<0, \forall \bar{x}(k) \in R^{n}$, from (13) we have $\forall A \in \mathcal{A}$

$$
\begin{array}{r}
\|Q \bar{x}(k+1)\|-\|Q \bar{x}(k)\|<0 \\
\|Q A \bar{x}(k)\|-\|Q \bar{x}(k)\|<0 \\
\|Q A S \bar{y}(k)\|-\|\bar{y}(k)\|<0
\end{array}
$$

where $\bar{y}(k)=Q \bar{x}(k)$.

Since (14) is valid $\forall \bar{y}(k) \neq 0$, we have

$$
\sup _{\bar{y}(k) \neq 0} \frac{\|Q A S \bar{y}(k)\|}{\|\bar{y}(k)\|}-1<0
$$

Hence, $\sigma\left(S^{-1} A S\right)<1 \forall A \in \mathcal{A}$.

Sufficiency: Let $S$ nonsingular be such that $\sigma\left(S^{-1} A S\right)<1 \forall A \in \mathcal{A}$. Then, choose $P=Q^{T} Q$ where $Q=S^{-1}$. Clearly, $P$ is positive definite. Then the claim is that the common quadratic Lyapunov function is $V(\bar{x})=\bar{x}^{T} P \bar{x}=\|Q \bar{x}(k)\|^{2}$. We prove the claim by showing that $\Delta V(\bar{x}(k))<0 \forall A \in \mathcal{A}$. This proof follows the same pattern as that in Theorem 4.2 by replacing the general induced norm there by the spectral norm $\sigma(\cdot)$.

Comment 2: It is evident that if there exists a matrix norm $\|\cdot\|$ such that $\|A\|<1 ; \forall A \in \mathcal{A}$; then, the switching system is stable, since the similarity transformation $S$ can be taken to be the identity matrix $I$. A convenient norm on the matrix is the spectral norm $\|\cdot\|_{\mathrm{sp}}$ equal to the largest singular value of the operand matrix. In view of the theorem and comment above, a simplified sufficiency condition, thus, is that $\sigma(A)<1 ; \forall A \in \mathcal{A}$ since $\sigma(\cdot)$ the spectral norm is the norm induced from the Euclidean vector norm.

Comment 3: In particular, this motivates interest in normal matrices as (for these matrices) eigen and singular values coincide. If $M$ is normal, $\rho(M)<1, \sigma(M)=\rho(M)<1$, thereby satisfying the condition mentioned in the above comment. A normal matrix $M$ is characterized by $M M^{*}=M^{*} M$ (see [1, Sec. 2.5]).

\section{SimultaneOUS NORMALIZATION}

Since the spectral radius is a norm on normal matrices and spectral radius of each individual Amatrix of the subsystems is necessarily less than unity, simultaneous transformation of $A_{i}, i=1 \cdots N$ into normal matrices through a similarity transformation is motivated. The following theorem is a fallout of the results in the previous section, and the proof follows from Theorem 4.1 and Comments 2 and 3 on Theorem 4.1.

Theorem 5.1 The switching system in (3) is stable if there exists nonsingular $S$ such that $S^{-1} A S$ is normal $\forall A \in \mathcal{A}$ and the spectral radius of each matrix $A$ is less than unity.

Since spectral radius of the matrices being less than unity is a necessary condition for stability, simultaneous similarity transformation of $A_{i}, i=1 \cdots N$ into normal matrices is considered. A matrix $A$ is said to be normalizable if there exists nonsingular $S$ such that $S^{-1} A S$ is normal. From [5], $S$ is necessarily of the form $S=T_{A} U$ where $T_{A}$ is a modal matrix of $A$ and $U$ is a unitary matrix. Let $\mathcal{N}_{A}=$ $\left\{S^{-1} A S: S=T_{A} U ; U\right.$ unitary $\}$. It can be shown that if $M \in \mathcal{N}_{A}$ then

$$
\mathcal{N}_{A}=\left\{U^{*} M U: U \text { is unitary }\right\} .
$$

Evidently, $\rho(A)=\rho(M) ; M \in \mathcal{N}_{A}$. To check for simultaneous normalization of the matrices $A_{i}, i=1 \cdots N$, initially, the problem of pairwise normalization of these matrices is considered. Once pairwise normalization is achieved, further conditions can be attached so that simultaneous normalization is achieved.

From now on, for simplicity, let the matrices $A_{i}, i=1 \cdots N$ have distinct eigen values. Then the modal matrix $T_{A}$ of $A$ can be represented as $\hat{T}_{A} K_{A} M_{A}$ where $\hat{T}_{A}$ is the modal matrix of $A$ with normalized columns of right eigen vectors of $A, K_{A}$ is a diagonal matrix with nonzero complex elements and is the scaling term and $M_{A}$ is a permutation matrix. Also, $M_{A} M_{A}^{T}=M_{A}^{T} M_{A}=I$. From [5, Theorem 4 and Corollary 1] we have the following.

Theorem 5.2: Let $A$ and $B$ be two normalizable matrices. Then they are simultaneously normalizable iff $T_{A} T_{A}^{*}=T_{B} T_{B}^{*}$. If $A$ and $B$ have distinct eigen values, then the above condition reduces to the existence of two positive definite matrices $D_{1}$ and $D_{2}$ such that

$$
\begin{array}{ll}
D_{1}=Q D_{2} Q^{*} & Q=\hat{T}_{A}^{-1} \hat{T}_{B} \\
D_{1}=K_{A} K_{A}^{*} ; & D_{2}=K_{B} K_{B}^{*} .
\end{array}
$$

Now a relationship is developed between pairwise normalization of the matrices $A_{i}, i=1 \cdots N$ and their simultaneous normalization. Let the matrices be pairwise normalizable and let the transformation $S_{i j}, i \neq j$ simultaneously normalize the matrices $A_{i}$ and $A_{j}, \forall i, j=1 \cdots N$. Also, $S_{i j}=S_{j i}, \forall i, j=$ $1 \cdots N$. The following theorem now gives the additional constraints on the transformations so that the matrices are simultaneously normalizable.

Theorem 5.3: A necessary and sufficient condition for simultaneous normalization of the matrices $A_{i}, i=1 \cdots N$ is 
that the matrices should be pairwise normalizable and there exist fixed $l_{1}, l_{2}$ in $1 \cdots N$ such that

$$
\begin{aligned}
& S_{l_{1} l_{2}}^{-1} S_{l_{1} i} \quad \text { or } \quad S_{l_{1} l_{2}}^{-1} S_{l_{2} i} \\
& \quad=U_{i} \text { a unitary matrix } \quad \forall i=1 \cdots N .
\end{aligned}
$$

Proof: If the matrices are simultaneously normalizable say by a transformation $W$ then clearly they are pairwise normalizable by the same transformation and, also, $W W^{-1}=$ $I$ is unitary. Hence, only sufficiency is to be proved.

The claim is that the transformation $S_{l_{1} l_{2}}$ simultaneously normalizes all the matrices. Now, since $S_{l_{1} i}$ and $S_{l_{2} i}$ normalize $A_{i}$, we have $\forall i=1 \cdots N$

$$
\begin{aligned}
S_{l_{1} l_{2}}^{-1} A_{i} S_{l_{1} l_{2}}= & S_{l_{1} l_{2}}^{-1} S_{l_{1} i} M_{A_{i}}^{1} S_{l_{1} i}^{-1} S_{l_{1} l_{2}} \text { or } \\
& S_{l_{1} l_{2}}^{-1} S_{l_{2} i} M_{A_{i}}^{2} S_{l_{2} i}^{-1} S_{l_{1} l_{2}} \\
= & U_{i} M_{A_{i}}^{1} U_{i}^{-1} \text { or } U_{i} M_{A_{i}}^{2} U_{i}^{-1} \text { from (17) } \\
= & \tilde{M}_{A_{i}} \in \mathcal{N}_{A_{i}} \text { from }(15)
\end{aligned}
$$

where $M_{A_{i}}^{1}, M_{A_{i}}^{2} \in \mathcal{N}_{A_{i}}$.

The condition in Theorem 5.3 involves checking for $S_{1}^{-1} S_{2}$ being unitary, where $S_{1}, S_{2}$ are two transformations that normalize the same matrix. The following theorem provides conditions on the transformations $S_{1}$ and $S_{2}$ for this to occur.

Theorem 5.4 Let $S_{1}$ and $S_{2}$ be two transformations that normalize a matrix $A$. Let $S_{i}=\hat{T}_{A} K_{i} M_{i} U_{i}, i=1,2$. Then, $S_{1}^{-1} S_{2}$ is unitary iff

$$
\left|\left(K_{1}\right)_{j j}\right|=\left|\left(K_{2}\right)_{j j}\right| ; \quad \forall j=1 \cdots n .
$$

Proof: Now

$$
\begin{aligned}
S_{1}^{-1} S_{2} & =\left(\hat{T}_{A} K_{1} M_{1} U_{1}\right)^{-1}\left(\hat{T}_{A} K_{2} M_{2} U_{2}\right) \\
& =U_{1}^{*} M_{1}^{T} D M_{2} U_{2}
\end{aligned}
$$

where $D=K_{1}^{-1} K_{2}$. Hence, we have

$$
\begin{aligned}
\left(S_{1}^{-1}\right. & \left.S_{2}\right)\left(S_{1}^{-1} S_{2}\right)^{*} \\
& =\left(U_{1}^{*} M_{1}^{T} D M_{2} U_{2}\right)\left(U_{1}^{*} M_{1}^{T} D M_{2} U_{2}\right)^{*} \\
& =U_{1}^{*} M_{1}^{T} D D^{*} M_{1} U_{1} \\
& =\tilde{U}_{1}^{*} D D^{*} \tilde{U}_{1}
\end{aligned}
$$

since $M_{1}$ is a permutation matrix. Now, $\tilde{U}_{1}^{*} D D^{*} \tilde{U}_{1}=I$ iff $D$ is also unitary or $D D^{*}=I$. Since $D=K_{1}^{-1} K_{2}, K_{1}, K_{2}$ diagonal, the condition that $D$ be unitary reduces to the one in (18).

The procedure of verifying the satisfiability of Theorem 5.1 is summarized below in two steps. Step 1) checks for pairwise normalization of the matrices as in [5], and Step 2) which is carried out upon the success of Step 1) checks for additional constraints on the similarity transformations $S_{i j}$ so that simultaneous normalization of the matrices is achieved.

Step 1: The matrices $A_{i}, i=1 \cdots N$ are checked for pairwise normalization. This is done as in [5]. The conditions for pairwise normalization of two matrices, say, $A_{l_{1}}$ and $A_{l_{2}}$ are exposited below. The condition (16) in Theorem 5.2 is to be verified, i.e., $K_{A_{l_{1}}}$ and $K_{A_{l_{2}}}$ are to be found such that $T_{A_{l_{1}}} T_{A_{l_{1}}}^{*}=T_{A_{l_{2}}} T_{A_{l_{2}}}^{*}$. As in Theorem 5.2, define $Q=\hat{T}_{A_{l_{1}}}^{-1} \hat{T}_{A_{l_{2}}}$. Compute the matrices $Q=\left[q_{k m}\right]$ and $Q^{*-1}=$ $\left[s_{k m}\right]$. With $K_{A_{l_{1}}}=\operatorname{diag}\left\{a_{k}\right\}, K_{A_{l_{2}}}=\operatorname{diag}\left\{b_{k}\right\}, D_{1}=$ $\operatorname{diag}\left\{\left|a_{k}\right|^{2}\right\}$ and $D_{2}=\operatorname{diag}\left\{\left|b_{k}\right|^{2}\right\}$, as in Theorem 5.2, the condition $D_{1}=Q D_{2} Q^{*}$ is valid iff

$$
\left|a_{k}\right|^{2} s_{k m}=q_{k m}\left|b_{m}\right|^{2} \quad \forall k, m=1 \cdots n .
$$

Now define the matrix $R_{l_{1} l_{2}}=\left[r_{k m}\right]$ for every nonzero $q_{k m}$

$$
r_{k m}=\frac{s_{k m}}{q_{k m}} \text {. }
$$

If the values undetermined from (20) can be adjusted such that $R_{l_{1} l_{2}}$ has rank one, then the matrices $A_{l_{1}}$ and $A_{l_{2}}$ are simultaneously normalizable.

Remark 1: A necessary condition for (19) to be satisfied is that $Q$ and $Q^{*-1}$ show the same zero-nonzero pattern and all nonzero entries satisfy argument $\left(s_{k m}\right)=\arg \left(q_{k m}\right)$.

Remark 2: If (19) is satisfied, then $R_{l_{1} l_{2}}$ can be written as

$$
r_{k m}=\frac{\left|b_{m}\right|^{2}}{\left|a_{k}\right|^{2}}, \quad \forall k, m=1 \cdots n .
$$

Hence, $R_{l_{1} l_{2}}$ is the outer product of two vectors whose $k$ th elements are $\left|b_{k}\right|^{2}=\left|\left(K_{A_{l_{2}}}\right)_{k k}\right|^{2}$ and $1 /\left|a_{k}\right|^{2}=1 /\left|\left(K_{A_{l_{1}}}\right)_{k k}\right|^{2}$. Hence, $R_{l_{1} l_{2}}$ is a positive rank one matrix. Also, if $R_{l_{1} l_{2}}$ is a rank one positive matrix then the system of equations in (19) have a solution.

Remark 3: Thus, if all the matrices are pairwise normalizable, let the transformation that pairwise normalizes $A_{i}$ and $A_{j}$ be

$$
S_{i j}=\hat{T}_{A_{i}} K_{i}(i j) M_{i}(i j) U_{i}(i j)=\hat{T}_{A_{j}} K_{j}(i j) M_{j}(i j) U_{j}(i j) .
$$

Then, as constructed in Step $1, R_{i j}$ corresponds to the outer product of the vectors whose $k$ th elements are $1 /\left|\left(K_{i}(i j)\right)_{k k}\right|^{2}$ and $\left|\left(K_{j}(i j)\right)_{k k}\right|^{2}$.

Remark 4: Equation (19) represents an overdetermined set of equations and does not uniquely determine the scaling matrices $K_{i}(i j)$ and $K_{j}(i j)$ to the extent of a scaling factor in the transformations $S_{i j}$ and, it is therefore necessary to check the conditions of Theorem 5.2 through the matrices $R_{i j}$.

Step 2: Once Step 1 is satisfied, the existence of the matrices $S_{i j}$ is ensured and the matrices $R_{i j}$ are determined as in Step 1. This step now checks for the satisfiability of (17) in Theorem 5.3. From Theorem 5.4 the condition (17) simplifies to the following:

$$
\begin{gathered}
\forall i=1 \cdots N, \exists l_{1}, l_{2} \ni\left|K_{l_{1}}\left(l_{1} l_{2}\right)\right|=\left|K_{i}\left(l_{1} i\right)\right| \text { or } \\
\left|K_{l_{2}}\left(l_{1} l_{2}\right)\right|=\left|K_{i}\left(l_{2} i\right)\right| .
\end{gathered}
$$

As in Remark 3 above, only the outer product of vectors formed out of these scaling matrices $K_{i}(i j)$ and $K_{j}(i j)$ is expressed as matrices $R_{i j}$. Hence, this condition simplifies to the following:

$$
\begin{aligned}
\forall i= & 1 \cdots N, \exists l_{1}, l_{2} \ni \text { the pair }\left(R_{l_{1} l_{2}}, R_{l_{1} i}\right) \\
& \left(R_{l_{2} l_{1}}, R_{l_{2} i}\right) \text { has a common premultiplying } \\
& \text { vector in the outer product representation. }
\end{aligned}
$$

This common premultiplying vector has the $j$ th element either $\left|\left(K_{l_{1}}\left(l_{1} l_{2}\right)\right)_{j j}\right|^{2}$ or $\left|\left(K_{l_{2}}\left(l_{1} l_{2}\right)\right)_{j j}\right|^{2}$ depending upon which of the two matrix pairs $\left(R_{l_{1} l_{2}}, R_{l_{1} i}\right)$ or $\left(R_{l_{2} l_{1}}, R_{l_{2} i}\right)$ satisfies (22). Appendix A provides a condition on two rank one 
matrices $P, Q$ which have a common premultiplying vector in their vector outer product representation. The Lemma and the remark that follows it in the Appendix can then be used to detect the existence of $l_{1}, l_{2}$ such that (22) is satisfied.

The example below illustrates the application of both the Steps 1 and 2 in checking for simultaneous normalization of the matrices $A \in \mathcal{A}$.

Example 5.1: Let $N=n=3$. Also let the matrices in $\mathcal{A}$ be

$$
A_{1}=\left[\begin{array}{lll}
-1.9 & 0.3 & 0.5 \\
-4.8 & 1.1 & 1.0 \\
-7.2 & 2.6 & 0.3
\end{array}\right] \quad A_{2}=\left[\begin{array}{ccr}
4.7 & -1.9 & -0.1 \\
-11.1 & -4.8 & 0.1 \\
-12.3 & -5.8 & 0.1
\end{array}\right]
$$

and

$$
A_{3}=\left[\begin{array}{lll}
1.7 & -0.433 & -0.033 \\
2.2 & -0.2 & -0.1 \\
1.4 & -0.5 & 0.6
\end{array}\right]
$$

It can be seen that $\rho\left(A_{1}\right)=0.781 ; \rho\left(A_{2}\right)=0.985 ; \rho\left(A_{3}\right)=$ 0.728 and are less than unity. Also, $\sigma\left(A_{1}\right)=9.327 ; \sigma\left(A_{2}\right)=$ $18.882 ; \sigma\left(A_{3}\right)=3.177$ are all greater than unity and, hence, the simplified sufficient condition in Comment 2 to Theorem 4.1 is not applicable. Now, pairwise normalization of these matrices is checked as in Step 1.

Step 1: The modal matrices of $A_{1}, A_{2}, A_{3}$ are, respectively

$$
\begin{aligned}
\hat{T}_{A_{1}} & =\left[\begin{array}{ccc}
0.229 & 0.33 / 0.689 & 0.333 /-1.69 \\
0.688 & 0.667 \overline{1 / .689} & -.667 \overline{\overline{-1.689}} \\
0.688 & 0.667 \overline{/ 2.33} & 0.667 \overline{/-2.33}
\end{array}\right] \\
\hat{T}_{A_{2}} & =\left[\begin{array}{ccc}
0.224 / 0.561 & 0.224 /-0.561 & 0.408 \\
0.57 \overline{\underline{0.364}} & 0.57 \overline{-0.364} & 0.817 \\
0.79 \overline{1 / 0.703} & 0.79 \overline{1 /-0.703} & 0.408
\end{array}\right]
\end{aligned}
$$

and

$$
\hat{T}_{A_{3}}=\left[\begin{array}{ccc}
0.341 /-1.82 & 0.341 / 1.18 & 0.218 \\
0.763 \overline{/-1.997} & 0.763 / 1.997 & 0.436 \\
0.55 \overline{/-2.337} & 0.55 \overline{/ 2.337} & 0.873
\end{array}\right] .
$$

To check for pairwise normalization of $A_{1}, A_{2}$, we find $Q_{12}=$ $\hat{T}_{A_{1}}^{-1} \hat{T}_{A_{2}}$ and $Q_{12}^{*-1}$, respectively, as

$$
\begin{aligned}
& {\left[\begin{array}{ccc}
0.689 /-0.225 & 0.689 / 0.225 & 0 \\
0.53 \overline{/-1.45} & 0.53 \overline{/ 2.14} & 0.685 /-1.226 \\
0.53 \overline{-2.14} & 0.53 \overline{/ 1.45} & 0.685 / 1.226
\end{array}\right]} \\
& {\left[\begin{array}{ccc}
0.726 \overline{-0.225} & 0.726 / 0.225 & 0 \\
0.47 \overline{1-1.45} & 0.47 \overline{1 / 2.14} & 0.73 /-1.226 \\
0.471 \underline{\overline{1-2.14}} & 0.47 \overline{11.45} & 0.73 \underline{3 / 1.226}
\end{array}\right] .}
\end{aligned}
$$

Hence, the matrix $R_{12}$ computed as in (20) and the entry $r_{13}$ not found from (20) is adjusted such that $R_{12}$ has unit rank is

$$
R_{12}=\left[\begin{array}{lll}
1.053 & 1.053 & 1.263 \\
0.889 & 0.889 & 1.067 \\
0.889 & 0.889 & 1.067
\end{array}\right]
$$

Similarly, $Q_{23}=\hat{T}_{A_{2}}^{-1} \hat{T}_{A_{3}}$ and $Q_{31}=\hat{T}_{A_{3}}^{-1} \hat{T}_{A_{1}}$ are computed. Also, $Q_{21}=Q_{12}^{-1}, Q_{13}=Q_{31}^{-1}, Q_{32}=Q_{23}^{-1}$ are computed. As before, the matrices $R_{23}, R_{21}, R_{32}$ and $R_{31}$ are calculated and the matrices $R_{13}$ and $R_{23}$ are

$$
\begin{aligned}
R_{13} & =\left[\begin{array}{lll}
1.132 & 1.132 & 1.105 \\
0.956 & 0.956 & 0.933 \\
0.956 & 0.956 & 0.933
\end{array}\right] \text { and } \\
R_{23} & =\left[\begin{array}{lll}
1.075 & 1.075 & 1.05 \\
1.075 & 1.075 & 1.05 \\
0.896 & 0.896 & 0.875
\end{array}\right] .
\end{aligned}
$$

It is seen that the matrices $R_{12}, R_{23}, R_{13}$ are of rank one and, hence, the matrices $A_{1}, A_{2}, A_{3}$ are pairwise normalizable. Also, the matrices $R_{21}, R_{32}, R_{31}$ are also of rank one.

Step 2: It is seen that the pair $\left(R_{12}, R_{13}\right)$ has a common premultiplying vector in their vector outer product representation. Thus, $l_{1}=1, l_{2}=2$ can be chosen and (22) is satisfied. Thus, the matrices are simultaneously normalizable and, hence, the corresponding switching system is stable.

Comment 4: Simultaneous normalization can be thought of as an extension of simultaneous diagonalization of the matrices since diagonal matrices are normal. This case has been studied in [6] as a class of commuting matrices and the proof there proceeds by actually constructing a common Lyapunov matrix. We show that this result follows naturally in our framework of simultaneously normalizable matrices. This ties the circle of ideas of demonstrating stability by showing the existence of or by actually constructing a common quadratic Lyapunov function for the matrices in $\mathcal{A}$.

Theorem 5.5 [6]: If the matrices $A_{i}, i=1 \cdots N$ commute pairwise, then the switching system (3) is stable.

Proof: Let the matrices $A_{1}, A_{2}, \cdots A_{n}$ commute pairwise. Then the matrices are simultaneously diagonalizable $[1$, Theorem 1.3.12] and, hence, simultaneously normalizable. The switching system then is evidently stable by Theorem 5.1.

To see this, the matrices are now pairwise diagonalizable [1, Theorem 1.3.12] and let $S_{i j}$ be the transformation which diagonalizes both $A_{i}$ and $A_{j}$. Here, $S_{i j}=S_{j i}$. The claim is that any of these $S_{i j}$ will simultaneously diagonalize all the matrices $A_{i}, i=1 \cdots N$. This is seen as shown below.

Now, if $S_{1}, S_{2}$ diagonalize a matrix $A$, and $D$ is any diagonal matrix, then $S_{1}^{-1} S_{2} D S_{2}^{-1} S_{1}=D$. This is because

$$
\begin{aligned}
S_{1}^{-1} S_{2} & =\left(\hat{T}_{A} K_{1} M_{1}\right)^{-1}\left(\hat{T}_{A} K_{2} M_{2}\right) \\
& =M_{1}^{T} D_{12} M_{2}, \quad \text { where } \\
D_{12} & =K_{1}^{-1} K_{2} \text { is diagonal. }
\end{aligned}
$$

Now

$$
\begin{aligned}
S_{1}^{-1} S_{2} D S_{2}^{-1} S_{1} & =M_{1}^{T} D_{12} M_{2} D M_{2}^{T} D_{12}^{-1} M_{1} \\
& =M_{1}^{T} D_{12} D D_{12}^{-1} M_{1} \\
& =D .
\end{aligned}
$$

Here, the notation is as previously used. $\hat{T}_{A}$ is the modal matrix of $A$ with normalized right eigen vectors of $A$ as its columns, $K_{1}, K_{2}$ are diagonal scaling matrices with nonzero complex entries, and $M_{1}, M_{2}$ are permutation matrices. Now, since $S_{l_{1} i}$ diagonalizes $A_{i}$ to say $D_{i}$, we have $\forall i=1 \cdots N$,

$$
\begin{aligned}
S_{l_{1} l_{2}}^{-1} A_{i} S_{l_{1} l_{2}} & =S_{l_{1} l_{2}}^{-1} S_{l_{1} i} D_{i} S_{l_{1} i}^{-1} S_{l_{1} l_{2}} \\
& =D_{i} \text { from(23). }
\end{aligned}
$$


Hence, any transformation $S_{l_{1} l_{2}}$ that diagonalizes $A_{l_{1}}$ and $A_{l_{2}}$ will also diagonalize the other matrices. Hence, the matrices are simultaneously diagonalizable.

\section{CONCLUSION}

Stability of the Sugeno-Takagi model [2] of a fuzzy system has been considered in the asymptotic sense. A necessary condition for the global asymptotic stability which generalizes a result reported in [3] is given. A sufficient condition is also provided here for the global asymptotic stability of the system. A particular case of this sufficient condition, simultaneous normalization of the system matrices, is considered and a constructive procedure to check for simultaneous normalization is developed. This condition is easily checked using software utilities such as Matlab. A necessary and sufficient condition for asymptotic stability is yet elusive and further efforts should be aimed in this direction. The approach considered in this paper concentrates only on the consequents of the fuzzy implication rules and brackets all antecedents together, i.e., these results hold for very general systems since stability is shown for all possible fuzzy sets and membership functions. A method to take into account the specific knowledge of antecedents is needed.

\section{APPENDIX A}

Given two rank one matrices $P$ and $Q$, a procedure is outlined below which checks whether the matrices have a common premultiplying vector in their vector outer product representation.
Lemma 1: Let $P=\left[p_{i j}\right]$ and $Q=\left[q_{i j}\right]$ be rank one matrices. Then necessary and sufficient conditions on the matrices so that they may have a common premultiplying vector in their vector outer product representation are

1) the zero-nonzero pattern of $P$ and $Q$ coincide. Upon a nonmatch, the column containing the zero that did not match should all be zero;

2) $\forall j=1 \cdots n$ :

$$
\forall i=1 \cdots n, \quad q_{i j} \neq 0, \quad \frac{p_{i j}}{q_{i j}}=k_{j} .
$$

Remark 1: If $P$ and $Q$ have a common premultiplying vector in their vector outer product representation, then $\exists a, b, c \in$ $R^{n} \ni P=a b^{T}$ and $Q=a c^{T}$. The two conditions in the lemma are evident from this representation.

\section{REFERENCES}

[1] R. A. Horn and C. R. Johnson, Matrix Analysis. Cambridge: Cambridge Univ. Press, 1985.

[2] T. Takagi and M. Sugeno, "Fuzzy identification of systems and its applications to modeling and control," IEEE Trans. Syst., Man, Cybern., vol. SMC-15, pp. 116-132, Jan./Feb. 1985.

[3] K. Tanaka and M. Sugeno, "Stability analysis and design of fuzzy control systems," Fuzzy Sets Syst., vol. 45, pp. 135-156, 1992.

[4] K. Tanaka and M. Sano, "A robust stabilization problem of fuzzy control systems and its application to backing up control of a truck-trailer," IEEE Trans. Fuzzy Syst., vol. 2, pp. 119-133, May 1994.

[5] C. Pommer and W. Kliem, "Simultaneously normalizable matrices," Linear Algebra Applicat., vol. 94, pp. 113-125, Sept. 1987.

[6] K. S. Narendra and J. Balakrishnan, "A common Lyapunov function for stable LTI systems with commuting A-matrices," IEEE Trans. Automat. Contr., vol. 39, pp. 2469-2471, Dec. 1994.

[7] P. Lancaster, Theory of Matrices. New York: Academic, 1969.

[8] T. Kailath, Linear Systems. Englewood Cliffs, NJ: Prentice-Hall, 1980.

[9] K. S. Tsakalis and P. A. Ioannou, Linear Time-Varying Systems-Control and Adaptation. Englewood Cliffs, NJ: Prentice-Hall, 1993. 